\title{
Ramsey interferometry of non-Hermitian quantum impurities
}

\author{
F. Tonielli $\odot,{ }^{1}$ N. Chakraborty $\odot,{ }^{2,3,4}$ F. Grusdt $\odot,{ }^{5,6}$ and J. Marino $\oplus^{7,8}$ \\ ${ }^{1}$ Institut für Theoretische Physik, Universität zu Köln, D-50937 Cologne, Germany \\ ${ }^{2}$ Centre for Advanced 2D Materials, National University of Singapore, 6 Science Drive 2, Singapore 117546 \\ ${ }^{3}$ Yale-NUS College, 16 College Avenue West, Singapore 138527 \\ ${ }^{4}$ Rudolf Peierls Centre for Theoretical Physics, Clarendon Laboratory, Parks Road, Oxford OX1 3PU, United Kingdom \\ ${ }^{5}$ Munich Center for Quantum Science and Technology (MCQST), Schellingstraße 4, D-80799 München, Germany \\ ${ }^{6}$ Fakultät für Physik, Ludwig-Maximilians-Universität, D-80799 München, Germany \\ ${ }^{7}$ Institut für Physik, Johannes Gutenberg Universität Mainz, D-55099 Mainz, Germany \\ ${ }^{8}$ Department of Physics, Harvard University, Cambridge, Massachusetts 02138, USA
}

(Received 15 March 2020; accepted 17 June 2020; published 1 July 2020)

\begin{abstract}
We introduce a Ramsey pulse scheme which extracts the non-Hermitian Hamiltonian associated with an arbitrary Lindblad dynamics. We propose a related protocol to measure via interferometry a generalized Loschmidt echo of a generic state evolving in time with the non-Hermitian Hamiltonian itself, and we apply the scheme to a one-dimensional weakly interacting Bose gas coupled to a stochastic atomic impurity. The Loschmidt echo is mapped into a functional integral from which we calculate the long-time decohering dynamics at arbitrary impurity strengths. For strong dissipation we uncover the phenomenology of a quantum many-body Zeno effect: Corrections to the decoherence exponent resulting from the impurity self-energy become purely imaginary, in contrast to the regime of small dissipation where they instead enhance the decay of quantum coherences. Our results illustrate the prospects for experiments employing Ramsey interferometry to study dissipative quantum impurities in condensed matter and cold-atom systems.
\end{abstract}

DOI: 10.1103/PhysRevResearch.2.032003

An atomic wire subject to localized particle losses, or a quantum spin chain subject to on-site dephasing, are instances of dissipative impurity problems. The nomenclature is borrowed from the traditional research field of equilibrium quantum impurities in many-body systems, which comprises archetypical cases ranging from $\mathrm{x}$-ray edge singularities to magnetic impurities embedded in fermions [1,2]. Systems with quantum impurities have represented important stepping stones in understanding the physics of strongly correlated systems, and by adding localized dissipation on an extended system, one could similarly gain insight in the mechanisms intertwining incoherent processes and quantum correlations in many-body systems.

The surge of interest in this modern area of research has been ignited by a few recent experiments in cold gases: Shining an electron beam on a localized spatial region of an atomic BEC of ${ }^{87} \mathrm{Rb}$ atoms [3-8] induces a Zeno effect which dictates that atom losses decrease at strong dissipation. Dissipative impurities can also constitute a resource in quantum many-body engineering, as they are employed to implement scanning gate microscopes of ultracold bosons $[9,10]$.

In its conventional formulation, the quantum Zeno effect predicts the freezing of the wave function when frequent

Published by the American Physical Society under the terms of the Creative Commons Attribution 4.0 International license. Further distribution of this work must maintain attribution to the author(s) and the published article's title, journal citation, and DOI. measurements exceed a rate threshold [11-18]. The phenomenon extends beyond the theory of quantum measurement, and it comprises the generic arrest of quantum evolution provided by stochastic fields [19], including the decoupling of a system from its decohering environment by the application of a sequence of fast pulses [20]. The connection between the Zeno effect and many-body physics has been first drawn in the context of quantum circuits where unitary gates and randomin-space and time projective measurements compete, inducing a transition in the entanglement entropy from volume to area law when measurements become frequent [21]. Primarily motivated by the experiments in Refs. [5-7], the physics of the Zeno effect has also entered the field of dissipative impurities. The effect of $1 / \omega$ noise on the transport properties of KaneFisher barriers $[22,23]$ have been studied with nonequilibrium Luttinger liquids [24], while a series of related works have shown that strong local losses can inhibit particles' emission at the Fermi surface [25-27]; the interplay of Zeno physics with many-body correlations has the potential to promote lossy mobile impurities into a novel class of Fermi polarons [28] and the list of examples could continue [29,30].

In general, the dynamics induced by a Markovian quantum master equation comprise an imaginary or non-Hermitian Hamiltonian (which is quadratic in the Lindblad operator) in combination with a term describing stochastic jumps driven by quantum noise [31-33]. In the following, we show that a sequence of Ramsey pulses can experimentally decouple these two contributions, and can be employed to measure via interferometry a generalized form of the Loschmidt echo, which evolves solely with the former. This protocol represents 

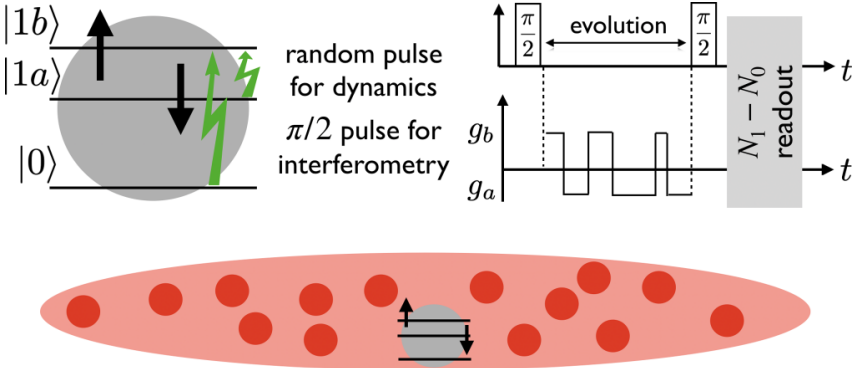

FIG. 1. An atomic impurity (gray dot) embedded in a onedimensional wire of cold bosons (in red). The impurity has three internal atomic levels: The level $|0\rangle$ is inert to scattering with the bosonic cloud, and is used as a control state for Ramsey interferometry which is employed to measure the Loschmidt echo for the non-Hermitian Hamiltonian associated with stochastic dynamics. The latter is generated by driving with a sequence of Markovian pulses the levels $|1 a\rangle$ and $|1 b\rangle$ which are density coupled to the background Bose gas.

an operative route to define non-Hermitian Hamiltonians and it could bring different experimental insight into communities focusing on this class of Hamiltonians; one example is the field of non-Hermitian topology [34-41] which is currently mostly implemented in photonics platforms [42].

We apply this scheme to the problem of a dissipative impurity in a weakly interacting Bose gas, and we predict, with functional integral techniques, a Zeno effect for the decohering exponent of the echo, which is nonperturbative in the impurity strength. This is at variance with previous works aiming at studying noise-averaged Loschmidt echoes in locally dephasing spin models $[43,44]$, which require the full Lindblad evolution, and it represents an alternative route to the study of dissipative impurities, since it does not rely on the measurement of transport properties across noisy barriers.

A general Markovian quantum master equation with one Lindblad channel reads

$$
i \partial_{t} \hat{\rho}=\hat{H}_{\text {eff }} \hat{\rho}-\hat{\rho} \hat{H}_{\text {eff }}^{\dagger}+\mathcal{L}_{\text {jump }}[\hat{\rho}],
$$

with the non-Hermitian Hamiltonian and quantum jump term being respectively

$$
\begin{aligned}
\hat{H}_{\mathrm{eff}} & =\hat{H}_{0}-i \gamma \hat{L}^{\dagger} \hat{L}, \\
\mathcal{L}_{\text {jump }}[\hat{\rho}] & =2 i \gamma \hat{L} \hat{\rho} \hat{L}^{\dagger} .
\end{aligned}
$$

The Ramsey protocol we propose to probe $\hat{H}_{\text {eff }}$ relies on the assumption that the strength of the interaction between the system and the environment can be controlled by an additional, discrete degree of freedom of the system. For simplicity, we consider a generic system with (at least) two internal states $|0\rangle$ and $|1\rangle$, the Lindblad dynamics being active only when the system is in the state $|1\rangle$. This is expressed by the following replacement, valid in the extended Hilbert space that includes the internal level,

$$
\hat{L} \rightarrow \hat{\tilde{L}}=\hat{L} \otimes|1\rangle\langle 1| \text {. }
$$

In cold atomic systems, the latter can be realized with spindependent interactions with the bath $[45,46]$ as employed in studies of Bose polarons [47-49] (see Fig. 1).
The density matrix of the system is prepared in a pure, factorized state $\hat{\tilde{\rho}}_{0}=|\psi\rangle\langle\psi|\otimes| 0\rangle\langle 0|$. A $\pi / 2$ pulse flips the internal state $|0\rangle \rightarrow(|0\rangle+|1\rangle) / \sqrt{2}$, and the full density matrix changes accordingly,

$$
\hat{\tilde{\rho}}_{0} \rightarrow \hat{\tilde{\rho}}_{1}=\frac{1}{2}\left(\begin{array}{ll}
|\psi\rangle\langle\psi| & |\psi\rangle\langle\psi| \\
|\psi\rangle\langle\psi| & |\psi\rangle\langle\psi|
\end{array}\right) .
$$

At this stage, the crucial observation is that, for a Lindblad operator as in Eq. (3), the quantum jump term acts only on the right-bottom element of the density matrix, i.e., on $\langle 1|\hat{\tilde{\rho}}(t)| 1\rangle$. In order to show that, we consider the action of the Liouvillian on product states of the form

$$
\tilde{\rho}=\sum_{n, m=0}^{1} p_{n m} \rho \otimes|n\rangle\langle m| .
$$

For Lindblad operators as in Eq. (3), the action from the left is nontrivial only on components with $n=1$,

$$
\tilde{L} \tilde{\rho}=(L \otimes|1\rangle\langle 1|) \tilde{\rho}=\sum_{m=0}^{1} p_{1 m} L \rho \otimes|n\rangle\langle m| .
$$

A similar equation holds for the action of $\tilde{L}^{\dagger}$ from the right, with the role of $n$ and $m$ being exchanged; the quantum jump term affects therefore only the component of $\tilde{\rho}$ with $n=1$, $m=1$,

$$
\mathcal{L}_{\text {jump }}[\tilde{\rho}]=2 \gamma p_{11} L \rho L^{\dagger} \otimes|1\rangle\langle 1| .
$$

The action of the full quantum master equation on the components of the density matrix reads therefore

$$
\begin{aligned}
i \partial_{t} \tilde{\rho}_{00} & =p_{00}\left(H_{0} \rho-\rho H_{0}\right), \\
i \partial_{t} \tilde{\rho}_{10} & =p_{10}\left(H_{\mathrm{eff}} \rho-\rho H_{0}\right), \\
i \partial_{t} \tilde{\rho}_{01} & =p_{01}\left(H_{0} \rho-\rho H_{\mathrm{eff}}^{\dagger}\right), \\
i \partial_{t} \tilde{\rho}_{11} & =p_{11}\left(H_{\mathrm{eff}} \rho-\rho H_{\mathrm{eff}}^{\dagger}+2 i \gamma p_{11} L \rho L^{\dagger}\right) .
\end{aligned}
$$

The combination of the second $\pi / 2$ pulse and of the contrast measurement probes only the nondiagonal components (8b) and (8c). In fact, a combination of a $\pi / 2$ pulse and a measurement of $\sigma_{z}$ is equivalent to a measurement of $\sigma_{x}$, since

$$
\left\langle\sigma_{z}\right\rangle=\operatorname{Tr}\left[\sigma_{z} R_{\pi / 2} \tilde{\rho}(t) R_{\pi / 2}^{\dagger}\right]=\operatorname{Tr}\left[R_{\pi / 2}^{\dagger} \sigma_{z} R_{-\pi / 2} \tilde{\rho}\right],
$$

where the matrix of a $\pi / 2$ pulse reads $R_{\pi / 2}=1 / \sqrt{2}\left(\sigma_{z}+\sigma_{x}\right)$, therefore $R_{\pi / 2}^{\dagger} \sigma_{z} R_{\pi / 2}=\sigma_{x}$. In conclusion, denoting by " $\operatorname{tr}$ " the trace acting only on the many-body degrees of freedom of the system, we get

$$
\begin{aligned}
\left\langle\sigma_{z}\right\rangle & =\operatorname{Tr}\left[\sigma_{x} \tilde{\rho}(t)\right]=\operatorname{tr}\left[\rho_{10}+\rho_{01}\right] \\
& =2 \operatorname{Re} \operatorname{tr}\left[p_{10} e^{i H_{0} t} e^{-i H_{\mathrm{eff}} t} \rho\right],
\end{aligned}
$$

which yields

$$
\left\langle\hat{\sigma}^{z}\right\rangle=\operatorname{Re}\left[\left\langle\psi\left|e^{i \hat{H}_{0} t} e^{-i\left(\hat{H}_{0}-i \hat{L}^{\dagger} \hat{L}\right) t}\right| \psi\right\rangle\right] .
$$

Such an implementation is inspired from the case of Hermitian Hamiltonians which we report for completeness in the Supplemental Material (SM [50]) (see also Refs. [51-54]).

Such a scheme can offer a systematic advantage over methods requiring averages over stochastic realizations, as it occurs 
in the evaluation of the conventional Loschmidt echo of noisedriven Hamiltonians. We finally notice that the derivation starting from Eq. (5) does not require us to restrict the initial state to a pure one, and would apply straightforwardly also to mixed states.

We now particularize our discussion to the setup of an ultracold one-dimensional Bose gas interacting with a localized atomic impurity. The discrete degree of freedom used for interferometry will be an internal state of the atom. To realize Eq. (3) and the Lindblad dynamics, the atom has a noninteracting internal level $|0\rangle$, and at least two additional levels, labeled as $|1 a\rangle$ and $|1 b\rangle$, density-coupled to bosons via the interaction Hamiltonian

$$
\hat{H}_{\text {int }}=\sum_{\sigma=a, b} g_{\sigma} \hat{n}_{\sigma}\left(x_{0}\right) \hat{n}_{B}\left(x_{0}\right),
$$

where $x_{0}$ denotes the position of the atom, $\hat{\psi}_{\sigma}$ its spinor wave function, $\hat{n}_{\sigma} \equiv \hat{\psi}_{\sigma}^{\dagger}\left(x_{0}\right) \hat{\psi}_{\sigma}\left(x_{0}\right)$ the occupation of the $\sigma$ level, and $\hat{n}_{B}\left(x_{0}\right)$ the bosonic number operator at $x=x_{0}$; we assume in the following $x_{0}=0$. Equation (12) describes an interaction whose strength is controlled by the states of the impurity. The Lindblad dynamics can be engineered by acting within the subspace $\{|1 a\rangle,|1 b\rangle\}$ with an additional external field, different from the one employed in the Ramsey protocol. With a sequence of $\pi$ pulses flipping between the two states, the coupling can be promoted to a time-dependent quantity $g(t)$, oscillating between $g_{a}$ and $g_{b}$, as depicted in Fig. 1. For a suitably chosen fast sequence of random pulses, $g(t)$ is a stochastic variable with first and second moments

$$
\langle g(t)\rangle=0, \quad\left\langle g(t) g\left(t^{\prime}\right)\right\rangle=\gamma \delta\left(t-t^{\prime}\right),
$$

where $\langle\cdots\rangle$ denotes the temporal average over several $\pi$ pulses. Higher-order moments are assumed to be negligible. The conditions on second and higher moments are equivalent to assume that the autocorrelation time of the density operator of the impurity is the smallest scale in the problem. The scheme proposed above, employing a fast sequence of random $\pi$ pulses, can be implemented in ultracold-atom systems: Rabi frequencies above $1 \mathrm{MHz}$ can be realized in alkali atoms, which leads to fast $\pi$ pulses compared to the typical atomic interaction energies. Meanwhile, couplings to other hyperfine states can be neglected due to large Zeeman splittings on the order of $100 \mathrm{MHz}$.

Our protocol can also be adapted to other experimental platforms, such as superconducting qubit arrays: A given subset of the Hilbert space can be driven with stochastic pulses, while the remaining states can be utilized for measurement purposes. The latter should be prone to dissipation, e.g., as a result of selection rules. Hence our scheme does not necessarily rely on the requirement that the dissipative channels support dark states.

The temporal average over multiple autocorrelation times yields an equation of motion for the density matrix equal to Eq. (1), with the Lindblad operator $\hat{\tilde{L}}=\hat{n}_{B}(0) \otimes|1\rangle\langle 1|$. Expanding the Bose field in terms of Bogoliubov excitations, the Lindblad operator and the non-Hermitian Hamiltonian (2) become respectively

$$
\hat{\tilde{L}} \simeq-\left(n_{0}+\sqrt{\frac{n_{0}}{2 \pi}} \int_{k} V_{k}\left(\hat{b}_{k}+\hat{b}_{k}^{\dagger}\right)\right) \otimes|1\rangle\langle 1|
$$

and

$$
\begin{aligned}
\hat{H}_{\mathrm{eff}}= & \hat{H}_{0}-i \gamma \hat{L}^{\dagger} \hat{L} \\
= & \int_{k} \omega_{k} \hat{b}_{k}^{\dagger} \hat{b}_{k}-i g\left(\rho^{2}+\tilde{\Lambda}_{\tau}\right) \\
& -2 i g \rho \int_{k} V_{k}\left(\hat{b}_{k}+\hat{b}_{-k}^{\dagger}\right) \\
& -i g \int_{k q} V_{k} V_{q}\left(2 \hat{b}_{k}^{\dagger} \hat{b}_{q}+\hat{b}_{k}^{\dagger} \hat{b}_{-q}^{\dagger}+\hat{b}_{k} \hat{b}_{q}\right) .
\end{aligned}
$$

where we have defined $\int_{k} \equiv \int_{-\Lambda_{\tau}}^{\Lambda_{\tau}} d k$. The cutoff $\Lambda_{\tau}$ is a consequence of the Markov approximation [cf. Eq. (13)] on the statistics of the $\pi$ pulses, which are assumed to evolve on the shortest timescale $\tau$ in the model. However, this assumption is no longer valid when the dispersion relation $\omega_{k}$ enters the particlelike regime and momenta are of the order of $k \simeq \Lambda_{\tau} \propto \sqrt{1 / \tau}$, thus requiring one to cut off momentum modes beyond this UV scale. The parameter $\tilde{\Lambda}_{\tau}$ in Eq. (15) comes from the normal ordering of $\hat{H}_{\text {eff }}$, and it is related to the cutoff $\Lambda_{\tau}$ via $\tilde{\Lambda}_{\tau}=\sqrt{2+\Lambda_{\tau}^{2}}-\sqrt{2}$.

In expression (15), $\hat{b}_{k}$ are the Bogoliubov annihilation operators in the BEC, $n_{0}$ is the density of the condensate, and we have defined

$$
\begin{aligned}
V_{k} & =\left(\frac{k^{2}}{2+k^{2}}\right)^{1 / 4}, \quad \omega_{k}=|k| \sqrt{1+k^{2} / 2}, \\
\rho & =\sqrt{2 \pi n_{0}}, \quad g=\frac{\gamma n_{0}}{4 \pi},
\end{aligned}
$$

where $g$ expresses the dissipation strength in $\hat{H}_{\text {eff }}$ and replaces the microscopic coupling constant (we have used units $\hbar=$ $c=m=\xi=1$, where $c$ is the speed of sound, $\xi$ the healing length, and $m$ the mass of the bosons).

The contrast (11) and the related Loschmidt amplitude $\mathcal{G}(t)$ are now expressed in terms of a functional integral with fixed boundary conditions in time, following the standard coherent state Trotter decomposition. The derivation follows Ref. [55], and it is discussed in detail in the Supplemental Material [50]. Specifically, from Eq. (15) we find

$$
\begin{aligned}
\mathcal{G}(t) & \equiv\left\langle 0\left|e^{-i t \hat{H}_{\mathrm{eff}}}\right| 0\right\rangle \\
& =\int_{b_{k}(0)=0}^{b_{k}(t)=0} D b D b^{*} \exp \left(i \int_{0}^{t} d s\left[\int_{k} \mathcal{A}_{k}\right]\right),
\end{aligned}
$$

where

$$
\mathcal{A}_{k} \equiv b_{k}^{*}(s)\left(i \partial_{s}-\omega_{k}\right) b_{k}(s)+i \gamma L^{*}(s) L(s) .
$$

We remark that the functional integral formula (18) is suited to describe the outcome of the interferometric measurement discussed above for any choice of Lindblad operator $\hat{L}$, which can be local or extended in space. Equation (18) is analogous to a Matsubara functional integral in imaginary time, as it can be readily seen from the similarity between the time evolution operator, $\exp (-i t \hat{H})$, and the Boltzmann weight, $\exp (-\beta \hat{H})$; accordingly, we define the real-time Matsubara frequencies $\omega_{n}=2 \pi n / t$, with $n \in \mathbb{Z}$. Implementing the boundary conditions requires, however, an additional Lagrange multiplier, as discussed in the Supplemental Material [50]. 


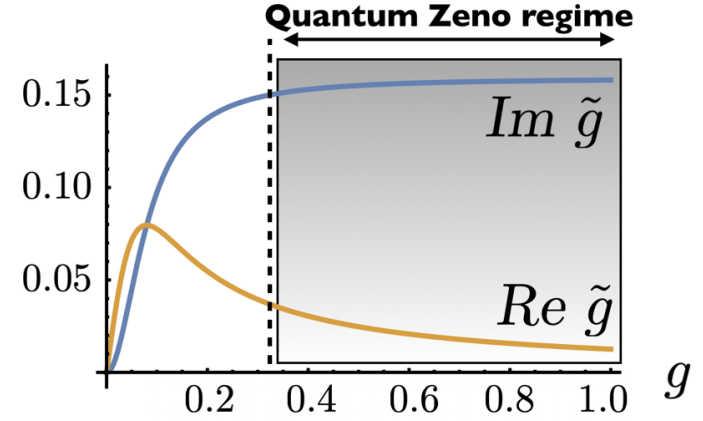

FIG. 2. Plot of the real and imaginary parts of the renormalized impurity strength $\tilde{g}$ as a function of the bare dissipation strength $g$. For $g \gg 1$ the real part vanishes, while the imaginary part reaches an asymptote at $1 / 2 \pi$, indicating the onset of a quantum Zeno regime.

The bare, $G_{k, n}^{0}$, and impurity dressed, $G_{k q, n}^{\text {eff }}$, Matsubara Green's functions can be derived from Eq. (18) after some manipulations which yield (see Supplemental Material [50] for the details of the calculations)

$$
\begin{aligned}
G_{k, n}^{0} & =\frac{2 V_{k}^{2} \omega_{k}}{\omega_{n}^{2}-\omega_{k}^{2}}, \\
G_{k q, n}^{\mathrm{eff}} & =G_{k, n}^{0} \delta_{k, q}-\frac{2 i g}{1+2 i g \int_{p} G_{p, n}^{0}} G_{k, n}^{0} G_{q, n}^{0} .
\end{aligned}
$$

Before proceeding further, we observe that Eq. (19b) carries crucial information on the perturbative expansion of $\log \mathcal{G}(t)$ in powers of the coupling $g$ : All corrections corresponding to a dressing of the Green's functions can be resummed and expressed in terms of a renormalized coupling strength

$$
\tilde{g} \equiv \frac{2 g}{1+2 i g \int_{p} G_{p, 0}^{0}}=\frac{2 g}{1-4 \pi i g} .
$$

This parameter is small since $|\tilde{g}|_{\max }=1 / 2 \pi \simeq 0.16$ (cf. Fig. 2); it is therefore convenient to develop an expansion of $\log \mathcal{G}$ in powers of $\tilde{g}$. The functional integral (18) can be now evaluated (see Supplemental Material [50]), obtaining the following exact expression of the Loschmidt amplitude,

$$
\begin{aligned}
\log \mathcal{G}(t)= & -g\left(\rho^{2}+\tilde{\Lambda}_{\tau}\right) t+2 i g^{2} \rho^{2} t \\
& \times\left(\int_{k, q} G_{k q, 0}^{\mathrm{eff}}+\operatorname{tr}\left[G_{0}^{\mathrm{eff}}\left(\sum_{n} G_{n}^{\mathrm{eff}}\right)^{-1} G_{0}^{\mathrm{eff}}\right]\right) \\
& +\frac{1}{2} \sum_{n} \operatorname{tr} \log \left[\left(G_{n}^{0}\right)^{-1} G_{n}^{\mathrm{eff}}\right] \\
& -\frac{1}{2} \operatorname{tr} \log \left[\left(\sum_{n} G_{n}^{0}\right)^{-1} \sum_{n} G_{n}^{\mathrm{eff}}\right] .
\end{aligned}
$$

In Eq. (21) the matrices act only in momentum space, and $G_{0}$ is shorthand for $G_{n=0}$; correspondingly, traces run only over momenta. We can observe here the role played by the renormalized coupling in the analytic expression. The first term in a naive perturbation theory corresponds to the first line, i.e., it is obtained by replacing $G^{\text {eff }} \rightarrow G^{0}$. Crucially, almost all corrections to naive perturbation theory are small, and under perturbative control even at strong coupling, since they can be resummed and expressed in terms of $\tilde{g}$, as manifested by the presence of the dressed Green's functions in Eq. (21). The only possibly relevant contribution to the naive perturbation theory comes from the second line, that also contains the bare coupling $g^{2}$ : The leading term at long times can be evaluated exactly, and the sum of the first and second line yields the Loschmidt echo

$$
\log \left[\mathcal{G}(t) / \mathcal{G}_{\tau}(t)\right] \simeq-\tilde{g} \rho^{2} t / 2,
$$

in terms of the amplitude $\mathcal{G}_{\tau}(t) \equiv \exp \left(-g \tilde{\Lambda}_{\tau} t\right)$, which can be controlled by shaping the noise profile. The right-hand side of Eq. (22) represents nonperturbative corrections to the leading decoherence damping $\mathcal{G}_{\tau}(t)$ expected in general for a stochastic scatterer embedded in an otherwise coherent medium.

Nevertheless, the renormalized coupling $\tilde{g}$, which is real for small values of the bare coupling $g$, becomes purely imaginary at strong bare coupling, $\tilde{g} \simeq i(2 \pi)^{-1}$, as illustrated in Fig. 2 . The fact that $\tilde{g}$ is imaginary for large dissipative strengths indicates that the rate decay function of the Loschmidt echo will be entirely dominated by the bare decay exponent $\propto g \tilde{\Lambda}$. The occurrence that all higher-order corrections to decoherence are neutralized and resummed to an imaginary exponent is an incarnation of the Zeno effect: For strong dissipation (large $g$ ), the incoherent scatterer perfectly reflects bosons which impinge upon it, and its only effect is to imprint a phase shift on reflected wave functions (see for related ideas the cold-atom experiment in Ref. [56]).

Conversely, in the conventional case of the Loschmidt echo of a decohering qubit coupled to an extended quantum system (e.g., a quantum spin chain), one expects a rate of decay which monotonously grows with the system bath coupling or with dissipation strength (see, for instance, Ref. [57]).

In conclusion, we have proposed how to measure, via Ramsey interferometry, the Loschmidt amplitude of an effective non-Hermitian Hamiltonian associated with a Lindbladian. The onset of a many-body Zeno effect can be directly probed by the readout of $\left\langle\sigma^{z}\right\rangle$ without resorting to measurements of transport properties or to probing unequal time correlation functions. We have demonstrated through Eqs. (19b) and (20) that unitarity is restored for quasiparticle dynamics at strong dissipation strength, while Eqs. (21) and (22) show that, in spite of the onset of the Zeno effect, a damping persists and becomes dominant at strong coupling. It would be interesting to study, in the future, whether the Zeno effects can manifest similarly in the interferometric properties of other systems, or whether their imprint on the Loschmidt echo is inherently nonuniversal.

Our results pave the way for a number of further exploratory directions. First, it would be natural to study an extension of our calculations in the case of a mobile impurity in view of recent connections between polarons and Zeno physics [28]. Furthermore, the approach developed for extracting the leading decay rate of $\mathcal{G}(t)$ is completely general, and it could be, for instance, extended to more realistic dissipative impurities by taking into account the spatial profile of the impurity wave function or the correlation time of the noise. Finally, the short-time pattern of the generalized 
Loschmidt echo could serve as a means to characterize the dynamical quantum phase transitions of non-Hermitian systems $[58,59]$. We also foresee the possibility of applying concepts developed for the study of dynamical topological phenomena $[59,60]$ to the more recent field of non-Hermitian topology $[61,62]$, with direct access to the echo of physically realizable non-Hermitian Hamiltonians defined via the protocol discussed here.

Since out-of-time order correlations can be measured via Ramsey interferometry [63], we also foresee in the future an extension of our results in the direction of probing scrambling in non-Hermitian quantum systems.

F.T. thanks C. Mordini for useful discussions. F.G. acknowledges support by the Deutsche Forschungsgemeinschaft (DFG, German Research Foundation) under Germany's Excellence Strategy - EXC-2111-390814868. J.M. was supported by the European Union's Horizon 2020 research and innovation programme under the Marie Sklodowska-Curie Grant Agreement No. 745608 (QUAKE4PRELIMAT).
[1] G. D. Mahan, Many-Particle Physics (Springer, Berlin, 2013).

[2] I. Affleck, in Exact Methods In Low-Dimensional Statistical Physics and Quantum Computing, edited by J. Jacobsen, S. Ouvry, V. Pasquier, D. Serban, and L. Cugliandolo, Lecture Notes of the Les Houches Summer School, LXXXIX, 2008 (Oxford University Press, Oxford, U.K., 2010), p. 3.

[3] T. Gericke, P. Würtz, D. Reitz, T. Langen, and H. Ott, Nat. Phys. 4, 949 (2008).

[4] V. A. Brazhnyi, V. V. Konotop, V. M. Pérez-García, and H. Ott, Phys. Rev. Lett. 102, 144101 (2009).

[5] D. A. Zezyulin, V. V. Konotop, G. Barontini, and H. Ott, Phys. Rev. Lett. 109, 020405 (2012).

[6] G. Barontini, R. Labouvie, F. Stubenrauch, A. Vogler, V. Guarrera, and H. Ott, Phys. Rev. Lett. 110, 035302 (2013).

[7] R. Labouvie, B. Santra, S. Heun, and H. Ott, Phys. Rev. Lett. 116, 235302 (2016)

[8] Y. S. Patil, S. Chakram, and M. Vengalattore, Phys. Rev. Lett. 115, 140402 (2015).

[9] M. Lebrat, S. Häusler, P. Fabritius, D. Husmann, L. Corman, and T. Esslinger, Phys. Rev. Lett. 123, 193605 (2019).

[10] L. Corman, P. Fabritius, S. Häusler, J. Mohan, L. H. Dogra, D. Husmann, M. Lebrat, and T. Esslinger, Phys. Rev. A 100, 053605 (2019).

[11] P. Facchi and S. Pascazio, Phys. Rev. Lett. 89, 080401 (2002).

[12] A. G. Kofman and G. Kurizki, Phys. Rev. A 54, R3750(R) (1996).

[13] A. G. Kofman, G. Kurizki, and T. Opatrný, Phys. Rev. A 63, 042108 (2001).

[14] A. Kofman and G. Kurizki, Nature (London) 405, 546 (2000).

[15] H. M. Wiseman and G. J. Milburn, Quantum Measurement and Control (Cambridge University Press, Cambridge, U.K., 2009).

[16] B. Misra and E. G. Sudarshan, J. Mater. Phys. 18, 756 (1977).

[17] W. M. Itano, D. J. Heinzen, J. J. Bollinger, and D. J. Wineland, Phys. Rev. A 41, 2295 (1990).

[18] M. C. Fischer, B. Gutiérrez-Medina, and M. G. Raizen, Phys. Rev. Lett. 87, 040402 (2001).

[19] T. Nakanishi, K. Yamane, and M. Kitano, Phys. Rev. A 65, 013404 (2001).

[20] P. Facchi, D. A. Lidar, and S. Pascazio, Phys. Rev. A 69, 032314 (2004).

[21] Y. Li, X. Chen, and M. P. A. Fisher, Phys. Rev. B 98, 205136 (2018).

[22] C. L. Kane and M. P. A. Fisher, Phys. Rev. Lett. 68, 1220 (1992).
[23] C. L. Kane and M. P. A. Fisher, Phys. Rev. B 46, 15233 (1992).

[24] E. G. Dalla Torre, E. Demler, T. Giamarchi, and E. Altman, Phys. Rev. B 85, 184302 (2012).

[25] H. Fröml, A. Chiocchetta, C. Kollath, and S. Diehl, Phys. Rev. Lett. 122, 040402 (2019).

[26] H. Fröml, C. Muckel, C. Kollath, A. Chiocchetta, and S. Diehl, Phys. Rev. B 101, 144301 (2020).

[27] S. Wolff, A. Sheikhan, S. Diehl, and C. Kollath, Phys. Rev. B 101, 075139 (2020).

[28] T. Wasak, R. Schmidt, and F. Piazza, arXiv:1912.06618.

[29] P. L. Krapivsky, K. Mallick, and D. Sels, arXiv:1911.08617.

[30] P. L. Krapivsky, K. Mallick, and D. Sels, J. Stat. Mech.: Theory Exp. (2019) 113108.

[31] H.-P. Breuer and F. Petruccione, The Theory of Open Quantum Systems (Oxford University Press, Oxford, U.K., 2002).

[32] U. Weiss, Quantum Dissipative Systems, Vol. 13 (World Scientific, Singapore, 2012).

[33] C. Gardiner and P. Zoller, Quantum Noise (Springer, Berlin, 1991).

[34] K. Esaki, M. Sato, K. Hasebe, and M. Kohmoto, Phys. Rev. B 84, 205128 (2011).

[35] S. Yao, F. Song, and Z. Wang, Phys. Rev. Lett. 121, 136802 (2018).

[36] M. S. Rudner and L. S. Levitov, Phys. Rev. Lett. 102, 065703 (2009).

[37] J. M. Zeuner, M. C. Rechtsman, Y. Plotnik, Y. Lumer, S. Nolte, M. S. Rudner, M. Segev, and A. Szameit, Phys. Rev. Lett. 115 , 040402 (2015).

[38] D. Leykam, K. Y. Bliokh, C. Huang, Y. D. Chong, and F. Nori, Phys. Rev. Lett. 118, 040401 (2017).

[39] S. Yao and Z. Wang, Phys. Rev. Lett. 121, 086803 (2018).

[40] H. Shen, B. Zhen, and L. Fu, Phys. Rev. Lett. 120, 146402 (2018).

[41] Z. Gong, Y. Ashida, K. Kawabata, K. Takasan, S. Higashikawa, and M. Ueda, Phys. Rev. X 8, 031079 (2018).

[42] T. Ozawa, H. M. Price, A. Amo, N. Goldman, M. Hafezi, L. Lu, M. C. Rechtsman, D. Schuster, J. Simon, O. Zilberberg, and I. Carusotto, Rev. Mod. Phys. 91, 015006 (2019).

[43] F. Tonielli, R. Fazio, S. Diehl, and J. Marino, Phys. Rev. Lett. 122, 040604 (2019).

[44] W. Berdanier, J. Marino, and E. Altman, Phys. Rev. Lett. 123, 230604 (2019).

[45] C. Chin, R. Grimm, P. Julienne, and E. Tiesinga, Rev. Mod. Phys. 82, 1225 (2010). 
[46] A. Amico, F. Scazza, G. Valtolina, P. Tavares, W. Ketterle, M. Inguscio, G. Roati, and M. Zaccanti, Phys. Rev. Lett. 121, 253602 (2018).

[47] N. B. Jørgensen, L. Wacker, K. T. Skalmstang, M. M. Parish, J. Levinsen, R. S. Christensen, G. M. Bruun, and J. J. Arlt, Phys. Rev. Lett. 117, 055302 (2016).

[48] M.-G. Hu, M. J. Van de Graaff, D. Kedar, J. P. Corson, E. A. Cornell, and D. S. Jin, Phys. Rev. Lett. 117, 055301 (2016).

[49] Z. Z. Yan, Y. Ni, C. Robens, and M. W. Zwierlein, Science 368, 190 (2020).

[50] See Supplemental Material at http://link.aps.org/supplemental/ 10.1103/PhysRevResearch.2.032003 for an overview of the interferometric scheme for Hamiltonians and for the details of the functional integral calculations.

[51] M. Knap, A. Shashi, Y. Nishida, A. Imambekov, D. A. Abanin, and E. Demler, Phys. Rev. X 2, 041020 (2012).

[52] J. Goold, T. Fogarty, N. Lo Gullo, M. Paternostro, and T. Busch, Phys. Rev. A 84, 063632 (2011).

[53] M. Knap, A. Kantian, T. Giamarchi, I. Bloch, M. D. Lukin, and E. Demler, Phys. Rev. Lett. 111, 147205 (2013).
[54] M. Cetina, M. Jag, R. S. Lous, I. Fritsche, J. T. Walraven, R. Grimm, J. Levinsen, M. M. Parish, R. Schmidt, M. Knap et al., Science 354, 96 (2016).

[55] A. Altland and B. D. Simons, Condensed Matter Field Theory (Cambridge University Press, Cambridge, U.K., 2010).

[56] N. Syassen, D. M. Bauer, M. Lettner, T. Volz, D. Dietze, J. J. Garcia-Ripoll, J. I. Cirac, G. Rempe, and S. Dürr, Science 320, 1329 (2008).

[57] D. Rossini, T. Calarco, V. Giovannetti, S. Montangero, and R. Fazio, Phys. Rev. A 75, 032333 (2007).

[58] M. Heyl, A. Polkovnikov, and S. Kehrein, Phys. Rev. Lett. 110, 135704 (2013).

[59] M. Heyl, Rep. Prog. Phys. 81, 054001 (2018).

[60] J. C. Budich and M. Heyl, Phys. Rev. B 93, 085416 (2016).

[61] E. J. Bergholtz, J. C. Budich, and F. K. Kunst, arXiv:1912.10048.

[62] K. Kawabata, K. Shiozaki, M. Ueda, and M. Sato, Phys. Rev. X 9, 041015 (2019).

[63] N. Y. Yao, F. Grusdt, B. Swingle, M. D. Lukin, D. M. Stamper-Kurn, J. E. Moore, and E. A. Demler, arXiv:1607.01801. 